\title{
Dynamical systems with multiplicative perturbations: the strong convergence of measures
}

\author{
by KATARZYNA HorbaCz (Katowice)
}

\begin{abstract}
We give sufficient conditions for the strong asymptotic stability of the distributions of dynamical systems with multiplicative perturbations. We apply our results to iterated function systems.

0. Introduction. We consider the effect of stochastic perturbation on discrete time multiplicative dynamical systems. For this purpose we study the behaviour of the sequence of distributions corresponding to a given system. Our aim is to establish simple criteria for the asymptotic stability of the distributions of the state variables.

In some aspects our definitions and criteria are similar to those in [3], [4] and [6]. There are, however, important differences. First, we prove the asymptotic stability of the distributions corresponding to a multiplicative dynamical system without the assumption that the perturbations have an absolutely continuous distribution. This was the main assumption in [3], [4].

In [6] the general case $x_{n+1}=T\left(x_{n}, \xi_{n}\right)$ is considered, with the sequence of perturbations $\xi_{n}$ having an arbitrary distribution. However, in this case the authors only prove that the sequence of distributions of $x_{n}$ is weakly convergent to a unique distribution.

We restrict ourselves to the case of multiplicative perturbations. We introduce the concept of the asymptotic stability of measures, and prove a sufficient stability criterion for strong asymptotic stability.

We use lower bound measure techniques [8]. Using this technique we study (in a particular case) the asymptotic behaviour of the Barnsley iterated function system [1], [2]. Iterated function systems are particularly useful in studying fractals.
\end{abstract}

1991 Mathematics Subject Classification: 47A35, 47D07,58F30.

Key words and phrases: dynamical system, Markov operator, strong asymptotic stability, iterated function system. 
1. Formulation of the problem. Let $m$ denote the standard Lebesgue measure on the $d$-dimensional Euclidean space $\mathbb{R}^{d}$. Let $V$ be a closed subset of $\mathbb{R}^{d}$ such that $m(V)>0$ and let $Y \subset \mathbb{R}^{k}$ be Borel measurable.

We consider the dynamical system

$$
x_{n+1}=S\left(x_{n}\right) \xi_{n} \quad \text { for } n=0,1, \ldots,
$$

where $S: V \rightarrow \mathbb{R}^{d k}$ is a Borel measurable transformation such that $S(V) \cdot Y$ $\subset V$ and the $\xi_{n}$ are $k$-dimensional random vectors with values in $Y$. We assume that the random vectors $\xi_{0}, \xi_{1}, \ldots$ are independent and identically distributed, i.e., the measure

$$
\varphi(A)=\operatorname{Prob}\left(\xi_{n} \in A\right) \quad \text { for } A \subset Y, A \text { a Borel set, }
$$

is the same for all $n$. We also assume that the initial value $x_{0}$ is a random vector independent of the sequence of perturbations $\left\{\xi_{n}\right\}$.

Our goal is the study of the asymptotic behaviour of the sequence $\left\{x_{n}\right\}$. Since the $\xi_{n}$ are random, the behaviour of $x_{n}$ is uncertain even with a specified $x_{0}$. Thus, we adopt the strategy of studying the sequence of distributions

$$
\mu_{n}(B)=\operatorname{Prob}\left(x_{n} \in B\right)
$$

where $B$ is a Borel subset of $V$.

The first step is to find a recurrence relation for the $\mu_{n}$. We have

$$
\mu_{n+1}(B)=E\left(1_{B}\left(x_{n+1}\right)\right), \quad B \subset V, B \text { a Borel set, }
$$

where $1_{B}$ denotes the characteristic (indicator) function of $B$. On the other hand, since $x_{n}$ and $\xi_{n}$ are independent, the expectation $E\left(1_{B}\left(x_{n+1}\right)\right)$ is evidently

$$
E\left(1_{B}\left(S\left(x_{n}\right) \xi_{n}\right)\right)=\int_{X} \int_{V} 1_{B}(S(x) y) \mu_{n}(d x) \varphi(d y) .
$$

It follows immediately that

$$
\mu_{n+1}(B)=\int_{X} \int_{V} 1_{B}(S(x) y) \mu_{n}(d x) \varphi(d y) .
$$

Thus, for a given initial measure $\mu_{0}$, evolution of the measures corresponding to the system (1) is described by the sequence of iterates $\left\{P^{n} \mu_{0}\right\}$, where

$$
P \mu(B)=\int_{X} \int_{V} 1_{B}(S(x) y) \mu(d x) \varphi(d y) .
$$

2. Strong asymptotic stability. In studying the asymptotic properties of the sequence of iterates $\left\{P^{n}\right\}$ it is convenient to introduce the definition of a Markov operator and asymptotic stability.

Let $(X, \mathcal{A})$ be a measure space with a $\sigma$-field $\mathcal{A}$. Denote by

$$
N(X)=(N(X, \mathcal{A}),\|\cdot\|)
$$


the linear space of all finite $\sigma$-additive functions on $X$ equipped with the norm $\|\nu\|=|\nu|(X)$; here $|\nu|=\nu^{+}+\nu^{-}$and

$$
\nu^{+}(A)=\nu(A \cap H), \quad \nu^{-}(A)=-\nu\left(A \cap H^{\prime}\right) \quad \text { for } A \in \mathcal{A}, \nu \in N(X),
$$

where $H, H^{\prime}$ is Hahn's decomposition of $X$ for $\nu$.

Let

$$
N_{+}(X)=\{\nu \in N(X): \nu \geq 0\}
$$

be the set of all finite measures on $(X, \mathcal{A})$,

$$
N_{\mathrm{p}}(X)=\left\{\nu \in N_{+}(X):\|\nu\|=1\right\}
$$

the set of all probability measures on $(X, \mathcal{A})$, and

$$
N_{\mathrm{a}}(X)=\{\nu \in N(X): \nu \ll m\}
$$

the set of all measures absolutely continuous with respect to the Lebesgue measure $m$.

A linear operator $P: N(X) \rightarrow N(X)$ is called a Markov operator if $P \nu \in N_{\mathrm{p}}(X)$ for $\nu \in N_{\mathrm{p}}(X)$. It is clear that the operator $P$ defined by (2) is a Markov operator.

We say that a Markov operator $P$ is strongly asymptotically stable if there exists a unique measure $\mu_{*} \in N_{\mathrm{p}}(X)$ such that $P \mu_{*}=\mu_{*}$ and

$$
\lim _{n \rightarrow \infty}\left\|P^{n} \mu-\mu_{*}\right\|=0 \quad \text { for } \mu \in N_{\mathrm{p}}(X) .
$$

In considering the asymptotic stability of the measures $P^{n} \mu$ we will use lower bound measure techniques.

A measure $\tilde{\mu} \in N_{+}(X)$ is called a lower measure for $P$ if

$$
\lim _{n \rightarrow \infty}\left\|\left(P^{n} \mu-\widetilde{\mu}\right)^{-}\right\|=0 \quad \text { for } \mu \in N_{\mathrm{p}}(X) .
$$

A lower measure $\widetilde{\mu}$ is called nontrivial if $\|\widetilde{\mu}\|>0$.

The importance of lower measures is a consequence of the following theorem:

THEOREM 1. A Markov operator $P$ is strongly asymptotically stable if and only if there is a nontrivial lower measure for $P$.

The proof may be found in [8].

Now we turn to the multiplicative dynamical system (1). Papers [3], [4] give sufficient conditions for asymptotic stability in terms of the evolution of densities under the assumption that the perturbations have an absolutely continuous distribution. However, by considering the recurrence relation for (1) we may also derive another sufficient condition for the asymptotic behaviour of the model system in terms of the convergence properties of measures. 
Theorem 1 will be our main tool in studying the asymptotic stability of the operator $P$ defined by (2). We start with a criterion for asymptotic stability in the case where $0 \in Y$ and $S: V \rightarrow \mathbb{R}^{d k}$ is arbitrary.

THEOREM 2. If the random vectors $\xi_{n}$ satisfy

$$
\varphi(\{0\})=\operatorname{Prob}\left(\xi_{n}=0\right)>0,
$$

then the operator $P$ given by (2) is strongly asymptotically stable.

Proof. Set $\eta=\varphi(\{0\})$ and consider the sequence $\mu_{n}=P^{n} \mu$ for a $\mu \in N_{\mathrm{p}}(V)$. By (2), we have

$$
\begin{aligned}
P \mu_{n}(B) & =\int_{X} \int_{V} 1_{B}(S(x) y) \mu_{n}(d x) \varphi(d y) \\
& \geq \int_{V} 1_{B}(0) \varphi(\{0\}) \mu_{n}(d x)=1_{B}(0) \eta \quad \text { for } B \subset V, B \text { a Borel set. }
\end{aligned}
$$

Defining the measure $\widetilde{\mu}$ by

$$
\widetilde{\mu}(B)= \begin{cases}\eta & \text { if } 0 \in B, \\ 0 & \text { if } 0 \notin B\end{cases}
$$

for $B$ a Borel set, we obtain $P^{n} \mu(B) \geq \widetilde{\mu}(B)$ for $n=1,2, \ldots$ Thus, $P$ has nontrivial lower measure $\widetilde{\mu}$, and by Theorem 1 the proof is complete.

Asymptotic stability of $P$ is in general more difficult to demonstrate in the case when the condition (4) is not satisfied. However, if $V=Y=[0,1]$ then the following theorem gives an answer to this problem.

TheOREM 3. Assume that $S:[0,1] \rightarrow[0,1]$ and the random variables $\xi_{n}$ have values in $[0,1]$. If there exist constants $\varepsilon \in(0,1]$ and $r>0$ such that

$$
\inf \{S(x): x \in[0, \varepsilon]\}>0
$$

and

$$
\varphi(A) \geq \operatorname{rm}(A)
$$

for every Borel sets $A \subset[0, \varepsilon]$, then the operator $P$ defined by (2) is strongly asymptotically stable.

Proof. Fix $\mu \in N_{\mathrm{p}}([0,1])$ and set $\mu_{n}=P^{n} \mu$ for $n=0,1 \ldots$ For every Borel set $B \subset[0,1]$ we have

$$
\begin{aligned}
\mu_{n+1}(B) & =\int_{[0,1]} \int_{[0,1]} 1_{B}(S(x) y) \mu_{n}(d x) \varphi(d y) \\
& \geq \int_{[0, \varepsilon]} \varphi\left(\frac{B}{S(x)} \cap[0,1]\right) \mu_{n}(d x)
\end{aligned}
$$




$$
\geq \mu_{n}([0, \varepsilon]) \inf _{x \in[0, \varepsilon]} \varphi\left(\frac{B}{S(x)} \cap[0,1]\right) .
$$

Set $\delta=\varphi([0, \varepsilon])$; according to (6) we have $\delta \geq r \varepsilon>0$. Furthermore,

$$
\begin{aligned}
\mu_{n}([0, \varepsilon]) & =\int_{[0,1]} \int_{[0,1]} 1_{[0, \varepsilon]}(S(x) y) \mu_{n-1}(d x) \varphi(d y) \\
& \geq \int_{[0,1]} \varphi([0, \varepsilon]) \mu_{n-1}(d x)=\delta .
\end{aligned}
$$

Setting $c=\inf _{x \in[0, \varepsilon]} S(x)$ and using (5)-(8) gives

$$
\begin{aligned}
\mu_{n+1}(B) & \geq \delta \inf _{x \in[0, \varepsilon]} \varphi\left(\frac{B}{S(x)} \cap[0,1]\right) \geq \delta \inf _{z \in[c, 1]} \varphi\left(\frac{B}{z} \cap[0, \varepsilon]\right) \\
& \geq \delta r \inf _{z \in[c, 1]} \frac{1}{z} m(B \cap[0, \varepsilon z]) \geq \delta r m(B \cap[0, \varepsilon c]) .
\end{aligned}
$$

Hence

$$
\widetilde{\mu}(B)=\delta r m(B \cap[0, \varepsilon c]) \quad \text { for } B \subset[0,1], B \text { a Borel set, }
$$

defines a nontrivial lower measure for $P$, which completes the proof.

3. Remarks. Now we consider connections between the strong asymptotic stability of measures and the asymptotic stability of densities.

Let $(X, \mathcal{A}, \lambda)$ be a $\sigma$-finite measure space. A linear operator $\bar{P}: L^{1}(X) \rightarrow$ $L^{1}(X)$ is called a Markov operator if $\bar{P}(D(X)) \subset D(X)$, where

$$
D(X)=\left\{f \in L^{1}(X): f \geq 0,\|f\|_{1}=1\right\}
$$

is the set of densities and $\|\cdot\|_{1}$ stands for the norm in $L^{1}(X)$.

We say that the Markov operator $\bar{P}: L^{1}(X) \rightarrow L^{1}(X)$ is asymptotically stable in $L^{1}(X)$ if there exists $f_{*} \in D(X)$ such that $\bar{P} f_{*}=f_{*}$ and

$$
\lim _{n \rightarrow \infty}\left\|\bar{P}^{n} f-f_{*}\right\|_{1}=0 \quad \text { for } f \in D(X) .
$$

Remark 1. Assume that the distribution $\varphi$ of the random vectors $\xi_{n}$ is absolutely continuous, $V=Y \subset \mathbb{R}$ and $S(x)>0$ for $x \in V$. Then for every initial distribution $\mu_{0}$ all the distributions $\mu_{n}$ with $n \geq 1$ are absolutely continuous. Denote by $g$ the density of $\varphi$. Then the density $f_{n}$ of $\mu_{n}$ is $f_{n}=\bar{P}^{n-1} f_{1}$ where

$$
f_{1}(x)=\int_{V} g\left(\frac{x}{S(y)}\right) \frac{1}{S(y)} 1_{V}\left(\frac{x}{S(y)}\right) \mu_{0}(d y)
$$

and

$$
\bar{P} f(x)=\int_{V} f(y) g\left(\frac{x}{S(y)}\right) \frac{1}{S(y)} 1_{V}\left(\frac{x}{S(y)}\right) d y, \quad f \in L^{1}(V) .
$$


Thus, if $\varphi$ is absolutely continuous we may state a sufficient condition for asymptotic stability in terms of the evolution of densities. This case was studied in [3], [4].

Further, if $\bar{P}$ is asymptotically stable in $L^{1}(V)$ then $P$ given by $(2)$ is strongly asymptotically stable and the sequence of measures $\mu_{n}=P^{n} \mu_{0}$ is strongly convergent to the absolutely continuous measure

$$
\mu_{*}(B)=\int_{B} f_{*}(x) d x, \quad B \subset V, B \text { a Borel set, }
$$

where $f_{*}$ is the stationary density of $\bar{P}$.

For $f \in L^{1}(X, \mathcal{A}, \lambda), f \geq 0$ we define

$$
\lambda_{f}(A)=\int_{A} f(x) \lambda(d x), \quad A \in \mathcal{A} .
$$

Remark 2. If the Markov operator $P: N(X) \rightarrow N(X)$ is strongly asymptotically stable and $P\left(N_{\mathrm{a}}(X)\right) \subset N_{\mathrm{a}}(X)$ then the operator $\bar{P}: L^{1}(X)$ $\rightarrow L^{1}(X)$ defined by $\bar{P} f=d\left(P \lambda_{f}\right) / d \lambda, f \in L^{1}(X)$, is asymptotically stable in $L^{1}(X)$.

4. Iterated function systems. In this section we study the strong asymptotic stability of an iterated function system [1], [2].

Consider $N$ given continuous transformations

$$
w_{i}: A \rightarrow A, \quad i=1, \ldots, N,
$$

on a closed set $A \subset \mathbb{R}^{d}$. Fix a probability vector $\left(p_{1}, \ldots, p_{N}\right)$ with $p_{i}>0$ and $\sum_{i=1}^{N} p_{i}=1$. Next choose $x_{0} \in A$ and define the sequence $\left\{x_{n}\right\}$ by successively choosing

$$
x_{n+1} \in\left\{w_{1}\left(x_{n}\right), \ldots, w_{N}\left(x_{n}\right)\right\} \quad \text { for } n=0,1, \ldots,
$$

in such a way that $x_{n+1}=w_{i}\left(x_{n}\right)$ with probability $p_{i}$.

We can easily reformulate the iterated function system of (10) within our framework. Assume that $Y$ is the set of all sequences $\{0, \ldots, 1, \ldots, 0\}$ where 1 is in the $i$ th place, $i=1, \ldots, N$. Further, consider a sequence of independent random vectors $\xi_{n}$ with values in $Y$ such that

$$
\operatorname{Prob}\left(\xi_{n}^{i}=1\right)=p_{i},
$$

where $\xi_{n}^{i}$ denotes the $i$ th coordinate of $\xi_{n}$. Define $S: A \rightarrow \mathbb{R}^{d N}$ by setting

$$
S(x)=\left(w_{1}(x), \ldots, w_{N}(x)\right) .
$$

Now,

$$
x_{n+1}=S\left(x_{n}\right) \cdot \xi_{n}
$$

gives the required sequence of random variables. 
In this case the operator $P$ governing the evolution of the measures corresponding to the iterated function system $(11),(12)$ is given by

$$
P \mu(B)=\sum_{i=1}^{N} p_{i} \int_{A} 1_{B}\left(w_{i}(x)\right) \mu(d x) \quad \text { for } \mu \in N(A) .
$$

Now consider the particular case when for some integer $i_{0} \in\{1, \ldots, N\}$ the function $w_{i_{0}}$ is constant on a set $B$.

Proposition 1. Assume that for some integer $i_{0} \in\{1, \ldots, N\}$ the function $w_{i_{0}}: A \rightarrow A$ is constant on some set $B \subset A$ of positive measure. Then the iterated function system (11), (12) is strongly asymptotically stable.

Proof. Choose $\mu \in N_{\mathrm{p}}(A)$ and set $\mu_{n}=P^{n} \mu$ for $n=0,1, \ldots$ From (13) we immediately obtain

$$
\begin{aligned}
\mu_{n+1}(E) & =\sum_{i=1}^{N} p_{i} \int_{A} 1_{E}\left(w_{i}(x)\right) \mu_{n}(d x) \\
& \geq p_{i_{0}} \int_{B} 1_{E}\left(w_{i_{0}}(x)\right) \mu_{n}(d x) \quad \text { for } E \subset A, E \text { a Borel set. }
\end{aligned}
$$

Since $w_{i_{0}}(x)=c$ for $x \in B$ where $c=\left(c_{1}, \ldots, c_{d}\right)$, we have

$$
\mu_{n+1}(E) \geq p_{i_{0}} 1_{E}(c) \mu_{n}(B) \text {. }
$$

Furthermore,

$$
\begin{aligned}
\mu_{n}(B) & =P^{n} \mu(B) \geq p_{i_{0}} \int_{A} 1_{B}\left(w_{i_{0}}(x)\right) \mu_{n-1}(d x) \\
& =p_{i_{0}} 1_{B}(c) \mu_{n-1}(A)=p_{i_{0}} 1_{B}(c) \quad \text { for } n=1,2, \ldots
\end{aligned}
$$

Thus, we obtain

$$
\mu_{n+1}(E) \geq p_{i_{0}}^{2} 1_{E \cap B}(c) \quad \text { for } E \subset A, E \text { a Borel set. }
$$

Hence the formula

$$
\widetilde{\mu}(E)= \begin{cases}p_{i_{0}}^{2} & \text { if } c \in E \cap B \\ 0 & \text { if } c \notin E \cap B\end{cases}
$$

for $E \subset A, E$ a Borel set, defines a nontrivial lower measure for $P$, and by Theorem 1 the proof is complete.

Next we consider the case where all transformations $w_{i}: A \rightarrow A$ are nonsingular, i.e. $m\left(w_{i}^{-1}(E)\right)=0$ whenever $m(E)=0$. It is evident that in this case the condition of Proposition 1 is not satisfied.

Since the operator $P$ corresponding to the iterated function system (11), 
(12) is given by

$$
P \mu(E)=\sum_{i=1}^{N} p_{i} \int_{A} 1_{E}\left(w_{i}(x)\right) \mu(d x), \quad E \subset A, E \text { a Borel set }
$$

or

$$
P \mu(E)=\sum_{i=1}^{N} p_{i} \mu\left(w_{i}^{-1}(E)\right)
$$

and the $w_{i}$ are nonsingular, we obtain

$$
P\left(N_{\mathrm{a}}(A)\right) \subset N_{\mathrm{a}}(A) .
$$

As a consequence, if $f=d \mu / d m$ then $\bar{P} f=d(P \mu) / d m$ is given by the formula

$$
\bar{P} f=\sum_{i=1}^{N} p_{i} P_{w_{i}} f
$$

where $P_{w_{i}}$ is the Frobenius-Perron operator corresponding to $w_{i}$.

Example 1. Let $A=[0,1]$. Consider

$$
w_{1}(x)=\left\{\begin{array}{ll}
\frac{x}{1-x} & \text { for } x \in\left[0, \frac{1}{2}\right), \\
2 x-1 & \text { for } x \in\left[\frac{1}{2}, 1\right],
\end{array} \quad w_{2}(x)=x \quad \text { for } x \in[0,1] .\right.
$$

We will show that for every probability vector $\left(p_{1}, p_{2}\right)$ with $0<p_{i}<1$, $i=1,2$, and $p_{1}+p_{2}=1$ the iterated function system (17) is not strongly asymptotically stable.

It is evident that $w_{1}$ and $w_{2}$ are nonsingular. Thus the operator $P$ corresponding to (17) given by

$$
P \mu(E)=p_{1} \mu\left(w_{1}^{-1}(E)\right)+p_{2} \mu\left(w_{2}^{-1}(E)\right)
$$

satisfies $P\left(N_{\mathrm{a}}([0,1])\right) \subset N_{\mathrm{a}}([0,1])$.

Assume that $P$ is strongly asymptotically stable. Then applying Remark 2 we obtain the asymptotic stability in $L^{1}([0,1])$ of the operator $\bar{P}$ given by

$$
\bar{P} f=p_{1} P_{w_{1}} f+p_{2} P_{w_{2}} f, \quad f \in L^{1}([0,1]) .
$$

Since

$$
P_{w_{1}} f(x)=\frac{1}{(1+x)^{2}} f\left(\frac{x}{1+x}\right)+\frac{1}{2} f\left(\frac{1}{2}+\frac{x}{2}\right), \quad P_{w_{2}} f(x)=f(x),
$$

we deduce that

$$
\bar{P} f(x)=p_{1}\left(\frac{1}{(1+x)^{2}} f\left(\frac{x}{1+x}\right)+\frac{1}{2} f\left(\frac{1}{2}+\frac{x}{2}\right)\right)+p_{2} f(x)
$$


is asymptotically stable in $L^{1}([0,1])$. Therefore there exists $f_{*} \in D([0,1])$ such that $\bar{P} f_{*}=f_{*}$, i.e.

$$
f_{*}(x)=p_{1} P_{w_{1}} f_{*}(x)+p_{2} f_{*}(x) .
$$

Thus there exists $f_{*} \in D([0,1])$ such that $P_{w_{1}} f_{*}=f_{*}$. But this is impossible, since the equation $P_{w_{1}} f=f$ has no solution in $L^{1}([0,1])$ except $f \equiv 0$ (see [7]).

Consequently the iterated function system (17) is not strongly asymptotically stable.

\section{References}

[1] M. F. Barnsley and S. Demko, Iterated function systems and the global construction of fractals, Proc. Roy. Soc. London Ser. A 399 (1985), 243-275.

[2] M. F. Barnsley, V. Ervin, D. Hardin and J. Lancaster, Solution of an inverse problem for fractals and other sets, Proc. Nat. Acad. Sci. U.S.A. 83 (1986), 19751977.

[3] K. Horbacz, Dynamical systems with multiplicative perturbations, Ann. Polon. Math. 50 (1989), 93-102.

[4] - Asymptotic stability of dynamical systems with multiplicative perturbations, ibid. 50 (1989), 209-218.

[5] A. Lasota and J. Tyrcha, On the strong convergence to equilibrium for randomly perturbed dynamical systems, ibid. 53 (1991), 79-89.

[6] A. Lasota and M. C. Mackey, Stochastic perturbation of dynamical systems: The weak convergence of measures, J. Math. Anal. Appl. 138 (1989), 232-248.

[7] —, - Probabilistic Properties of Deterministic Systems, Cambridge Univ. Press, Cambridge 1985.

[8] M. Podhorodyński, Stability of Markov processes, Univ. Iagell. Acta Math. 27 (1988), 285-296.

INSTITUTE OF MATHEMATICS

SILESIAN UNIVERSITY

BANKOWA 14

40-007 KATOWICE, POLAND 